\title{
Comunicação
}

[Communication]

\section{Ocorrência de neoplasias em cães e gatos da mesorregião metropolitana de Belém, PA entre 2005 e 2010}

\author{
[Occurrence of neoplasms in canine and feline from the Metropolitan Mesoregion \\ of Belém, PA from 2005 to 2010] \\ A.P.S. Priebe ${ }^{1}$, G. Riet-Correa ${ }^{1}$, L.J.A. Paredes ${ }^{2}$, M.S.F. Costa ${ }^{2}$, \\ C.D.C. Silva ${ }^{1}$, M.B. Almeida ${ }^{1}$ \\ ${ }^{1}$ Programa de pós-graduação em Saúde Animal na Amazônia - UFPA, PA \\ ${ }^{2}$ Aluno de graduação - Faculdade de Medicina Veterinária - UFPA, PA
}

\begin{abstract}
Neoplasia é uma entidade patológica caracterizada por um conjunto de fenômenos biológicos com origem no núcleo das células. Fatores como a nutrição balanceada, vacinação, métodos de diagnóstico e protocolos terapêuticos, cada vez mais específicos e eficazes, contribuem para a maior longevidade de cães e gatos (Maria et al., 1998). Por outro lado, observa-se elevação na incidência de doenças crônicas, como é o caso das neoplasias (Richards et al., 2001).
\end{abstract}

Embora nas regiões Sul, Sudeste, Centro-oeste e Nordeste os dados sobre a epidemiologia das neoplasias em pequenos animais estejam bem consolidados, o mesmo não ocorre na região Norte do país, onde ainda não existem trabalhos publicados sobre esse assunto. Este trabalho teve o objetivo de relatar a ocorrência de neoplasias em cães e gatos domésticos da mesorregião metropolitana de Belém. Foram avaliadas biópsias de tumores provenientes de clínicas e hospitais veterinários da mesorregião metropolitana de Belém, no período de 2005 a 2010. A mesorregião metropolitana de Belém é composta pelos municípios de Ananindeua, Barcarena, Belém, Benevides, Bujaru, Castanhal, Inhangapi, Marituba, Santa Bárbara do Pará, Santa Isabel do Pará e Santo Antônio do Tauá, com cerca de 2.467.828 habitantes

As neoplasias foram classificadas de acordo com suas características histológicas predominantes, segundo a classificação de tumores em animais

Recebido em 27 de abril de 2011

Aceito em 9 de setembro de 2011

E-mail: begeres@yahoo.com.br domésticos da Organização Mundial da Saúde, publicado pela Armed Forces Institute of Pathology (Misdorp et al., 1999)

Foram estudadas 226 biópsias de tumores, sendo 193 amostras de cães $(85,3 \%)$ e 33 de gatos $(14,7 \%)$. A idade de aparecimento dos tumores foi informada em 184 fichas e variou de oito meses a 20 anos de idade. A média de idade em cães e gatos ficou em 8,8 e 8,9 anos, respectivamente. Outros estudos retrospectivos demonstraram tendência de aumento do número de neoplasias em função do avanço da idade (De Nardi et al., 2002), com decréscimo em animais extremamente idosos (Misdorp, 1990).

Dados quanto ao sexo foram informados em 188 fichas. As fêmeas foram mais afetadas, representando $83 \%$ dos caninos e $70 \%$ dos felinos. Em Curitiba, PR, 69,7\% dos animais eram fêmeas, entretanto, quando se excluíram as neoplasias de mama, as fêmeas representaram $53 \%$ do total de diagnósticos (De Nardi et al., 2002).

Em cães, 91 eram neoplasias de pele (47\%), 74 eram de mama (38\%), oito do sistema reprodutor (4\%), oito da cavidade oral (4\%), quatro de ossos e cartilagens $(2 \%)$, três oculares $(2 \%)$, três do sistema digestório (2\%) e duas do sistema urinário (1\%). O sistema tegumentar tem se mostrado o principal sítio de desenvolvimento de neoplasias em cães (Souza et al., 2001; Dobson et al., 2002). Em felinos, 29 eram neoplasias da glândula mamária $(88 \%)$, duas da pele $(6 \%)$, uma do sistema respiratório (3\%) e uma da 
cavidade oral $(3 \%)$. Dentre todas as neoplasias diagnosticadas, $75 \%(171 / 226)$ eram malignas, e $25 \%(55 / 226)$ benignas. A demora na apresentação dos pacientes ao serviço veterinário pode ter sido a responsável pelo maior índice de malignidade, pois tumores benignos podem vir a se tornar malignos com o tempo (Sonremno et al., 2009).

Dos 103 tumores de mama, 74 foram diagnosticados em cães $(72 \%)$ e 29 em gatos (28\%), e a maioria das neoplasias eram malignas, compreendendo $84 \%$ dos casos. Resultados semelhantes foram encontrados na literatura nacional (Oliveira Filho et al., 2010).

Os tipos histológicos das neoplasias mamárias diagnosticados em cadelas foram 0 carcinossarcoma (24\%), carcinoma túbulopapilar simples $(22,5 \%)$, carcinoma complexo $(18,5 \%)$, carcinoma sólido $(12,7 \%)$, tumor misto benigno (12\%), adenoma complexo $(2,5 \%)$, fibrossarcoma $(2,5 \%)$, adenoma simples $(2,5 \%)$, carcinoma não infiltrativo $(1,4 \%)$ e carcinoma mucinoso $(1,4 \%)$. O carcinoma simples está entre as neoplasias mamárias mais comumente diagnosticadas em cadelas (Salvado, 2010), seguido dos tumores mistos maligno e benigno (Oliveira Filho et al., 2010). Em gatas, os tipos histológicos encontrados foram carcinoma túbulo papilar simples $(44,7 \%)$, carcinoma sólido $(34,5 \%)$, carcinoma em tumor misto benigno (7\%), adenoma simples (7\%), tumor misto benigno $(3,4 \%)$ e hiperplasia ductal $(3,4 \%)$.

As neoplasias de pele representaram $41,1 \%$ (93/226) do número total de neoplasias, sendo o mastocitoma $(11,8 \%)$ e o carcinoma de células escamosas (CCE) (100\%) os tumores de pele mais frequentes em cães e gatos, respectivamente. $\mathrm{Na}$ pele e glândulas anexas foram diagnosticados 28 padrões histológicos diferentes em cães, nominalmente: mastocitoma $(11,8 \%)$, tricoblastoma meduzoide $(9,7 \%), \mathrm{CCE}$ $(8,6 \%)$, melanoma $(8,6 \%)$, carcinoma apócrino $(7,5 \%)$, adenoma perianal $(6,5 \%)$, epitelioma sebáceo (5,5\%), papiloma $(5,5 \%)$, hemangiossarcoma $(3,2 \%)$, lipossarcoma bem diferenciado $(3,2 \%)$, tumor de células basais (3,2\%), tricoepitelioma (3,2\%), hemangiopericitoma $(2,1 \%)$, adenoma sebáceo $(2,1 \%)$, plasmocitoma anaplásico $(2,1 \%)$, hemangioma $(2,1 \%)$, lipoma $(2,1 \%)$, fibrossarcoma $(2,1 \%)$ e linfoma cutâneo não epiteliotrópico (2,1\%). Diagnosticaram-se, também, carcinoma de glândulas ceruminosas, carcinoma de células basais, cisto infundibular, osteossarcoma extraesquelético, cistoadenoma apócrino, pilomatricoma, ceratose actínica e carcinoma das glândulas de Meibomian, com um caso cada. O mastocitoma também foi a neoplasia de pele mais frequente em outros estudos epidemiológicos recentes (Meirelles et al., 2010). Nos felinos, o CCE foi a única neoplasia diagnosticada durante o período de estudo. O CCE representa $15 \%$ dos tumores em felinos e 5\% em caninos, segundo Vail e Withrow (2007).

O sistema reprodutor representou $4 \%$ do número total de neoplasias diagnosticadas em cães (8/193), sendo o tumor venéreo transmissível (TVT) a neoplasia mais frequente desse sistema, com 50\% (4/8) do total de amostras. Os demais diagnósticos foram: leimioma uterino, leiomiossarcoma uterino, cistoadenoma de ovário e seminoma. Dados semelhantes foram encontrados por Souza et al. (2001), em cujo estudo o TVT foi a neoplasia mais diagnosticada, seguida pelo leiomioma e seminoma.

Foram registrados oito casos de neoplasias orais, representando $4 \%$ da amostra total. Entre as neoplasias da cavidade oral diagnosticaram-se melanoma $(37,5 \%)$, epulide fibromatoso $(25 \%)$, CCE (25\%) e fibrossarcoma de boca $(12,5 \%)$. Em um estudo retrospectivo de neoplasias orais em cães, as mais frequentemente diagnosticadas foram papiloma, melanoma, fibrossarcoma, epulis acantomatoso, CCE e epulide fibromatoso (Salgado et al., 2008). Somente um gato foi diagnosticado com CCE.

O tecido ósseo e cartilaginoso representou $2 \%$ (4/226) do total de amostras estudadas, sendo todos da espécie canina. Os padrões histológicos foram: hemangiossarcoma ósseo, condroma ósseo primário, osteossarcoma osteoblástico e osteossarcoma pouco diferenciado. Dobson et al. (2002) encontraram 3,2\% de frequências dessas neoplasias.

Todas as neoplasias oculares foram diagnosticadas em caninos, sendo $1,5 \%$ da amostra total (3/226). Diagnosticou-se um adenoma do corpo ciliar, um adenoma das glândulas sebáceas tarsais e um adenocarcinoma da glândula da terceira pálpebra. Dobson et al. 
(2002) verificaram a frequência de $0,2 \%$ $(5 / 2546)$ de neoplasias oculares.

O sistema digestório foi representado por três amostras, sendo um carcinoma pancreático exócrino, um adenoma de reto e um adenocarcinoma de reto. $\mathrm{O}$ adenoma de reto e o adenocarcinoma de reto são os tipos histológicos mais comumente encontrados no reto de cães, segundo Sobral et al. (2009).

No sistema urinário dos cães, foram diagnosticados dois tipos histológicos distintos: carcinoma de células transicionais e hemangiossarcoma de bexiga. A bexiga é o órgão mais acometido por neoplasias desse sistema (Carvalho e Brum, 2009).

As neoplasias de pele foram as mais diagnosticadas nos cães, entretanto, nos gatos, prevaleceram as da glândula mamária. A idade média de aparecimento das neoplasias nas duas espécies foi de aproximadamente 8,8 anos. A ocorrência dos diferentes tipos de neoplasias, nas duas espécies, foi semelhante ao encontrado na maioria das publicações nacionais e internacionais.

Palavras-chave: cão, gato, ocorrência, neoplasias

\begin{abstract}
Data on the occurrence of cancers in dogs and cats from the Metropolitan Mesoregion of Belém are not available in the literature. This paper describes the occurrence of neoplasms in these species from 2005 to 2010. The canine cutaneous system was the most affected in this study (47\%), followed by the mammary gland (39\%), reproductive system (4\%), oral cavity (4\%), bones and joints (2\%), ocular system $(1.5 \%)$, digestive system $(1.5 \%)$ and urinary tract (1\%). In feline species, the mammary gland accounted for $87.8 \%$, followed by the cutaneous system (6\%), respiratory system (3\%) and oral cavity (3\%).
\end{abstract}

Keywords: dog, cat, occurrence, neoplasms

\section{AGRADECIMENTOS}

À Fundação de Amparo a Pesquisa do Estado do Pará (FAPESPA).

\section{REFERÊNCIAS BIBLIOGRÁFICAS}

CARVALHO, M.B.; BRUM, A.M., Neoplasias do sistema urinário In: DALECK, C.R.; DE NARDI, A.B.; RODASKY, S. Oncologia em cães e gatos. São Paulo: Roca, 2009. p.386-398.

DE NARDI, A.B.; RODASKI, S.; SOUSA, R.S. et al. Prevalência de neoplasias e modalidades de tratamentos em cães atendidos no hospital veterinário da Universidade Federal do Paraná. Arch. Vet. Sci., v.7, p.15-26, 2002.

DOBSON, J.M.; SAMUEL, S.; MILSTEIN, H. et al. Canine neoplasia in the UK: estimates of incidence rates from a population of insured dogs. J. Small Anim. Pract., v.43, p.240-246, 2002.
MARIA, P.P.; SOBRAL, R.A.; DALECK, C.R. Casuística de cães portadores de neoplasias atendidos no Hospital Veterinário da Unesp/Jaboticabal durante o período de 01/01/95 a 01/05/97, In: CONGRESSO BRASILEIRO DE CIRURGIA E ANESTESIOLOGIA VETERINÁRIA, 3., 1998, Belo Horizonte. Resumo... Belo Horizonte: UFMG, 1998. p.61.

MEIRELLES, A.E.W.B.; OLIVEIRA, E.C.; RODRIGUES, B.A. et al. Prevalência de neoplasmas cutâneos em cães da região metropolitana de Porto Alegre, RS: 1017 casos (2002 a 2007). Pesq. Vet. Bras., v30, p.968-973, 2010 .

MISDORP, W.; ELSE R.W.; HELLMÉN, E. et $a l$. Histological classification of the mammary tumors of the dog and the cat In: WORLD Health Organization International Histological Classification of Tumors of Domestic Animals. 2.ed. Washington: Armed Forces Institute of Pathology, 1999. p.11-58. 
MISDORP, W. General considerations In: MOULTON, J.E. (Ed.). Tumors in domestic animals. 3.ed., Berkeley: University of California, 1990. p.1-22.

OLIVEIRA FILHO, J.C.; KOMMERS, G.D.; MASUDA, E.K. et al. Estudo retrospectivo de 1.647 tumores mamários em cães. Pesqui. Vet. Bras., v.30, p.177-185, 2010.

RICHARDS, H.G.; McNEIL, P.E.; THOMPSON, H. et al. An epidemiological analysis of a caninebiopsies database compiled by a diagnostic histopathology service. Prev. Vet. Med., v.51, p.125-136, 2001.

SALGADO, B.S.; FERREIRA, T.S.; CARVALHO, G.D. et al. Estudo retrospectivo das neoplasias orais de cães atendidos no Departamento de Veterinária da Universidade Federal de Viçosa. Vet. Zootec., v.15, p.12-14, 2008.

SALVADO, I. Estudo retrospectivo das neoplasias em canídeos e felídeos domésticos, analisadas pelo laboratório de anatomia patológica da Faculdade de Medicina Veterinária da Universidade Técnica de Lisboa, no período compreendido entre 2000 e 2009. 97f. 2010. Dissertação (Mestrado em Medicina Veterinária) - Faculdade de Medicina Veterinária, Universidade Técnica de Lisboa, Lisboa.
SOBRAL, R.A.; DALECK, C.R.; RODASKI, S. et al. Neoplasias do sistema digestório In: DALECK, C.R.; DE NARDI, A.B.; RODASKY, S. Oncologia em cães e gatos. Brasil: ROCA. 2009. p. 318-326.

SORENMO, K.U.; KRISTIANSEN, V.M.; COFONE, M.A. et al. Canine mammary gland tumors; a histological continuum from benign to malignant; clinical and histopathological evidence. Vet. Comp. Oncol., v.7, p.162-172, 2009.

SOUZA, V.T.F.; PARAGUASSU, A.A.; MOREIRA, E.L.T. Ocorrência de neoplasias em caninos na cidade de Salvador, Bahia (Achados de biópsias). Rev. Bras. Saúde Prod. Anim., v.2, p.53-58, 2001.

VAIL, D.M.; WITHROW, S.J. Tumors of the skin and subcutaneous tissues. In: WITHROW, S.J.; VAIL, D.M. Small animal clinical oncology. 4.ed. Canada: Saunders, 2007, p.382. 